\title{
Wide Dynamic Range Germanium Detector for Perturbation Crystallography
}

\author{
H. Graafsma, P. Thorander, G. W. J. C. Heunen and J. Morse \\ European Synchrotron Radiation Facility, Avenue des Martyrs, 38043 Grenoble, France
}

(Received 11 March 1996; accepted 18 April 1996)

\begin{abstract}
An IR detector based on a cooled germanium photodetector has been tested for applications in X-ray diffraction. The detector can be used simultaneously in photon-counting mode and current mode

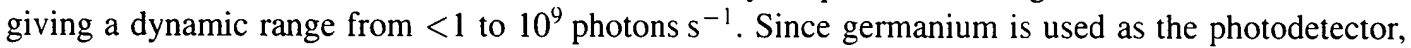
its efficiency at energies above $25 \mathrm{keV}$ is much better than the silicon equivalents. The detector proved to be highly linear both in the low-flux region $\left(<10^{5}\right.$ photons $\left.\mathrm{s}^{-1}\right)$ where photon counting is used and in the high-flux region $\left(>10^{5}\right.$ photons $\left.\mathrm{s}^{-1}\right)$ where the detector is run in current mode. The response time of the detector is of the order of $1 \mu \mathrm{s}$, making it suitable for studies in perturbation crystallography, especially when coupled to a lock-in amplifier. As an example, the shift of a reflection of $\mathrm{LiNbO}_{3}$ induced by an external electric field was determined with the germanium detector and lock-in amplifier.
\end{abstract}

\section{Keywords: perturbation crystallography; X-ray detectors; large dynamic range; germanium photodiodes.}

\section{Introduction}

The development of the third-generation synchrotron sources has given a dramatic increase in brilliance. Thanks to new developments in X-ray optics this increased brilliance is to a great extent conserved during beam conditioning, resulting in a much higher photon flux on the sample. In a range of experiments, the dynamic range and count-rate capability of the detector have now become limiting factors. This is especially true for perturbation studies where generally one has strong reflections since large and almost perfect crystals are used and the accuracy is limited by counting statistics (Paturle, Graafsma, Sheu, Coppens \& Becker, 1991; Graafsma et al., 1992). Since large samples, often with heavy elements, are used, a relatively high photon energy is needed in order to reduce absorption effects. In this case Si photodiodes, although also having a large dynamic range (Jemian, 1990; Storb, Dedek, Weber, Lengeler \& Schuster, 1991), have too low a detection efficiency.

Another type of experiment where the detector has become a limiting factor is the study of modulated structures or crystals with pseudo symmetry, where the satellite reflections are many orders weaker than the main reflections. In order to measure the main and satellite reflections without heavily attenuating the beam for the main reflections, a detector with a large dynamic range is needed.

Therefore, in order to fully profit from the increased brilliance of the new X-ray sources, it is necessary to develop new detectors with a larger dynamic range, higher counting capabilities and good detection quantum efficiencies for medium to high photon energies.

\section{Description of the unit}

The detector unit (403HS, Applied Detector Corporation) consists of a compact cylindrical liquid-nitrogen cryostat which houses a cooled $50 \mathrm{~mm}^{2}$ high-purity germanium crystal and preamplifier. The crystal thickness is $5 \mathrm{~mm}$ permitting high detection efficiencies for photon energies between 10 and $100 \mathrm{keV}$. The detector can be operated in either horizontal or inclined configurations and has a liquidnitrogen hold time of approximately $8 \mathrm{~h}$ and weighs $2.7 \mathrm{~kg}$. The detector was originally developed for IR measurements in a 'current mode' of operation in conjunction with a 'chopped' signal source and synchronous lock-in signal detection. At our request, the manufacturer replaced the standard sapphire IR window with an X-ray transparent beryllium window for these tests.

The preamplifier is a classic charge-feedback design, with a frequency response set by the feedback elements consisting of a $1 \mathrm{M} \Omega$ resistance in parallel with a $0.5 \mathrm{pF}$ capacitor. In the 'current integration' mode of operation the voltage generated across the feedback resistance by the mean signal current limits the maximum measurable signal at an X-ray flux equivalent of $\sim 2 \times 10^{10}$ photons s$^{-1}$ for $10 \mathrm{keV} \mathrm{X}$-rays, and $2 \times 10^{9}$ photons $^{-1}$ for $100 \mathrm{keV}$ $\mathrm{X}$-rays. The practical lower frequency limit of operation is set by the 'd.c.' drift of the preamplifier around a nominal value of $-1 \mathrm{~V}$; this drift arises principally from the variation in the operating point of the input-junction field-effect transistor of the preamplifier and leakage current variations across the detector crystal itself. After an initial cool-down period of $1 \mathrm{~h}$, the d.c. drift is $<5 \mu \mathrm{V} \mathrm{min}{ }^{-1}$. To put this figure in perspective, note that a steady-state

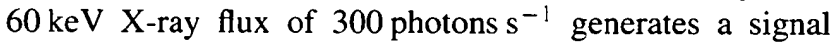


of $1 \mu \mathrm{V}$. The measured root-mean-square signal noise for an averaging period of $1 \mathrm{~s}$ is also $1 \mu \mathrm{V}$ for the detector. If used to measure low X-ray fluxes $\left(<10^{5}\right.$ photons $\left.\mathrm{s}^{-1}\right)$ the detector may be operated in a photon-counting mode. A good signal spectrum with a $3 \mathrm{keV}$ FWHM noise figure is obtained from a $59.5 \mathrm{keV}{ }^{241} \mathrm{Am}$ source when the output of the detector is post-amplified (Tennelec TC244, $2 \mu \mathrm{s}$ shaping time, $10^{3}$ photons $\mathrm{s}^{-1}$ ) and fed into a multichannel analyzer (Fig. 1). Note that pole zero compensation cannot be achieved with a standard nuclear spectroscopy amplifier, and this results in degradation of the spectrum at high count rates. Using a simple single-channel discriminator and operating the detector in a single-channel counting mode eliminates the problems of drift associated with the current mode of signal measurements, and assuming measurements with X-ray energies above $20 \mathrm{keV}$, the only significant detector noise is that arising from X-ray background in the experimental hutch.

\section{Linearity test}

The linearity of the unit has been tested both in the low-flux region, where photon counting is used, and in the high-flux region, where the current mode is used.

The linearity in the low count-rate region was tested at a rotating-anode source with Mo target $(17.45 \mathrm{keV})$. The incident flux was controlled by regulating the current setting of the generator. The a.c. output of the germanium detector was amplified by a factor of 1250 , using an ORTEC 575A; the signal was subsequently treated by a single-channel analyzer (ORTEC 550A) in order to eliminate electronic noise. The flux was measured both with the germanium detector and with an NaI scintillator (BEDE). The dead time of the NaI scintillator was calibrated before using the method of Chipman (1969), allowing for the exact

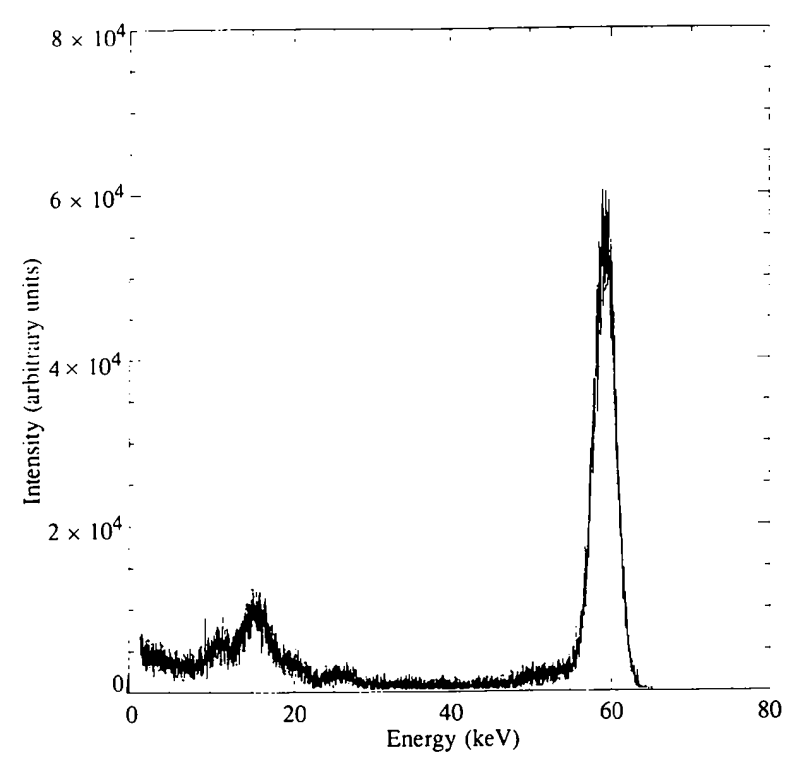

Figure 1

Spectrum of an ${ }^{241} \mathrm{Am}$ source recorded with the germanium detector and a multichannel analyzer. The americium peak at $59.5 \mathrm{keV}$ has a FWHM of $3 \mathrm{keV}$. determination of the true incoming number of photons. Fig. 2 shows the number of counts $s^{-1}$ recorded with the germanium detector as a function of true incoming number of photons $\mathrm{s}^{-1}$ determined with the calibrated $\mathrm{NaI}$ scintillator. The solid curve gives the calculated response of the detector using a non-paralyzable dead time $\tau$ of $1.9 \mu \mathrm{s}$ using (1) (Chipman, 1969),

$$
N_{t}=N_{r} /\left(1-N_{r} \tau\right)
$$

where $N_{r}$ is the recorded photon flux, $N_{t}$ the true incoming photon flux, and $\tau$ the dead time of the detector. It should be pointed out that both (1) and the dead time $\tau$ depend on the time structure of the source used, and can be significantly different when used at the ESRF in 1/3 filling mode (see Fig. $4 a$ ). It is seen that the unit can reliably be used up to a flux of $1.5 \times 10^{5}$ photons $\mathrm{s}^{-1}$, which is comparable with a standard $\mathrm{NaI}$ detector.

The linearity measurements for the high count-rate mode were made at the Materials Sciences beamline (ID11/BL2) of the European Synchrotron Radiation Facility (Kvick \& Wulff, 1992). A monochromatic beam of $22 \mathrm{keV}$ was used. The relatively low energy was selected in order to be able to make a good comparison between the voltage given by the germanium detector and the flux measured with a calibrated Si photodiode. The Si diode was read out by a Keithley 486 picoammeter, and the germanium detector by a Keithley K2001 multimeter. Fig. 3(a) shows the recorded voltage given by the germanium detector as a function of incoming number of photons. It is seen that the unit shows excellent linearity up to $10^{9}$ photons $\mathrm{s}^{-1}$ at $22 \mathrm{keV}$; above this value the preamplifier saturates. In Fig. 3(b), the voltage output of the detector in the lower-flux region is given. The figure shows that the voltage can reliably be used down to $3 \times 10^{4}$ photons $\mathrm{s}^{-1}$ at $22 \mathrm{keV}$, while the photon-

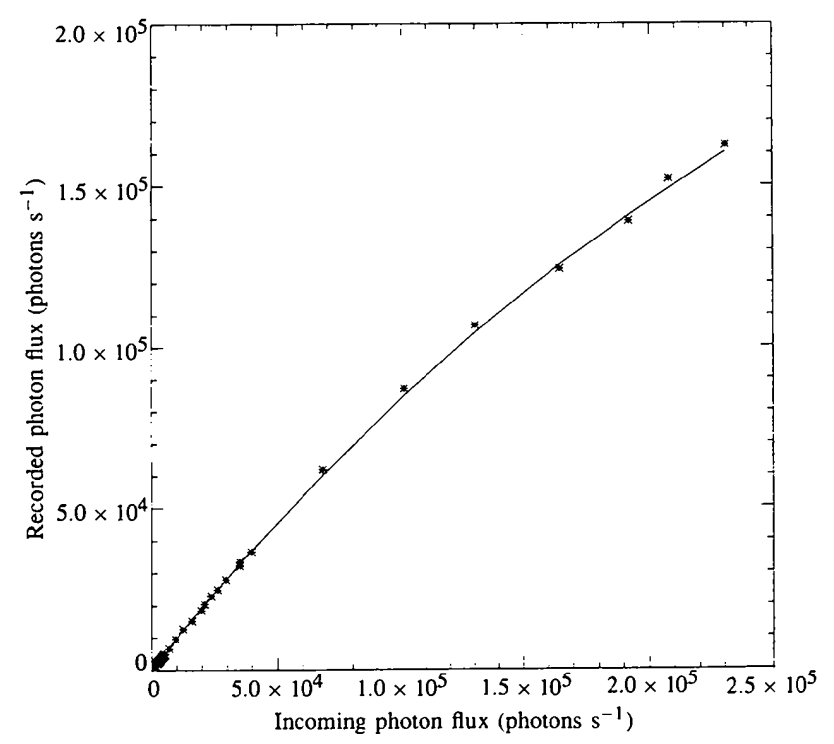

Figure 2

Recorded flux as a function of incoming photon flux, using the photon-counting mode of the germanium detector. The solid curve shows the calculated response of the detector using a dead time of $1.9 \mu \mathrm{s}$. 
counting mode can be used up to $1.5 \times 10^{5}$ photons $\mathrm{s}^{-1}$. Since both modes are used simultaneously and no switching between the two modes is needed, both output signals can be recorded during a single scan and the region between $3 \times 10^{4}$ and $1.5 \times 10^{5}$ photons $\mathrm{s}^{-1}$ can be used to scale the two modes together.

\section{Time response}

Since the detector is to be used in studies where the response of a crystal to an external perturbation is determined, its time response is an important characteristic besides maximum equivalent count rate and dynamic range. The time response of the detector was tested using the time structure of the X-ray beam of the ESRF, which for $1 / 3$ filling mode is given in Fig. 4(a). Fig. 4(b) shows the response of the germanium detector recorded with a digital storage oscilloscope. The germanium detector is fast enough to see the 'super bunches', but not fast enough to separate the single bunches within the super bunch. The $1 \mu$ s response time is in agreement with the RC time constant of the preamplifier and the pulse duration in counting mode. It should be noted that in order to use the detector in 'current mode' at the ESRF running in $1 / 3$ or hybrid mode, low-pass filtering is used in order to average

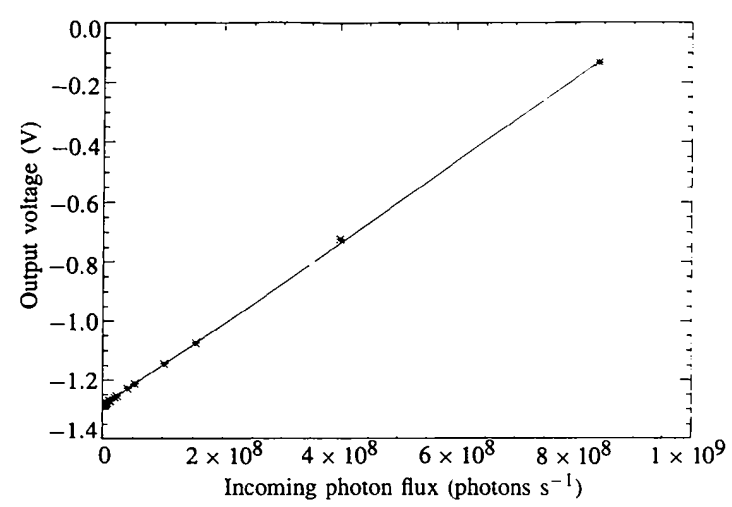

(a)

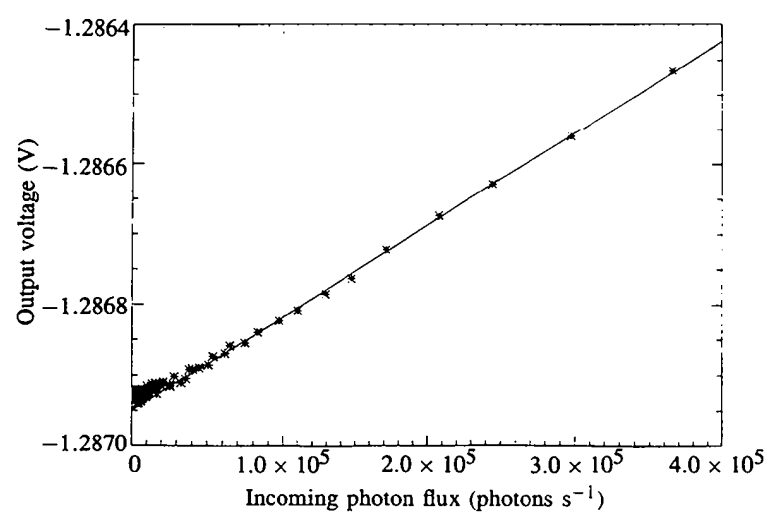

(b)

Figure 3

Output voltage of the germanium detector as a function of incoming photon flux (22 keV): $(a)$ the high-flux range, and $(b)$ an enlargement of the low-flux range. The solid line shows a fit of a straight line to the data points. over the super bunches. This low-pass filter is chosen such as to average over the super bunches while being fast enough not to average over the perturbation applied to the sample.

\section{Crystals in electric fields}

Since the germanium detector gives a voltage output it can be readily used with a lock-in amplifier to determine small changes in the diffracted signal in perturbation measurements. The shifts of the diffraction peaks of an $\mathrm{LiNbO}_{3}$ crystal in an external electric field were measured (Fujimoto, 1982; Ståhl, Kvick \& Abrahams, 1990) using the germanium detector and a digital lock-in amplifier (SRS model 850). The quasi-static electric field was applied in two steps of plus and minus $700 \mathrm{~V}$. giving an electric field of $3.8 \times 10^{6} \mathrm{~V} \mathrm{~m}^{-1}$, with a frequency of $33 \mathrm{~Hz}$. The measurements were performed at a rotating-anode source (Mo radiation).

Fig. 5(a) shows a step scan of the (0012) reflection where at each point of the scan the diffracted signal corresponding to both the plus and minus field is recorded with an $\mathrm{NaI}$ scintillation detector and home-built synchronization electronics (Paturle et al., 1991). The shift of the peak due to the piezoelectric effect is clearly visible. The dashed curve in Fig. 5(b) gives the difference between the plus and minus signal. The solid line in Fig. $5(b)$ gives the change in the diffraction profile determined with the germanium detector and lock-in amplifier. At each step of the scan a single reading was taken, with the integration time of the lock-in

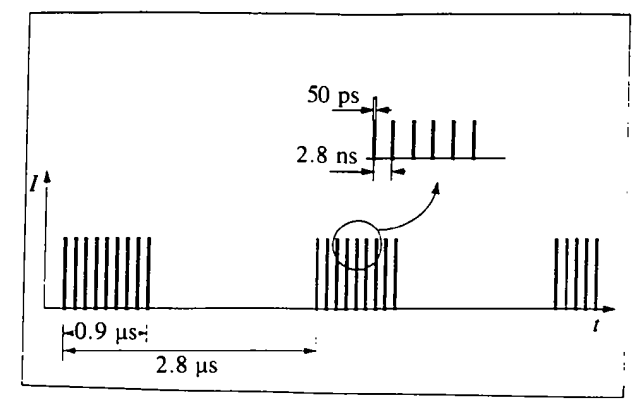

(a)

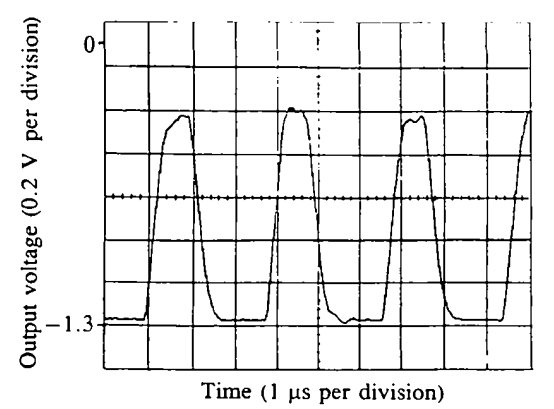

(b)

Figure 4

(a) Time structure of the ESRF in 1/3 filling mode, 1/3 of the ring is filled with 331 bunches, and $2 / 3$ of the ring ( 661 bunches) is empty. (b) Response of the germanium detector recorded with a storage oscilloscope. 
amplifier set to $300 \mathrm{~ms}$. It is seen that the two results are in good agreement.

Fig. $5(b)$ also shows that with the lock-in amplifier differences down to 1000 photons $\mathrm{s}^{-1}$ at $17.45 \mathrm{keV}$ can be detected using a time constant of $300 \mathrm{~ms}$. This limit can be significantly reduced by increasing the integration time of the amplifier. This will, of course, increase the data-acquisition time per point and thus prolong the total scanning time per profile.

It should be noted that when using a lock-in amplifier, only information about the difference between the two states is obtained (Fig. $5 b$ ), but no information about the individual peaks (Fig. $5 a$ ). To overcome this problem an $\mathrm{Si}$ diode used in transmission can be mounted in front of the germanium detector in order to determine the average

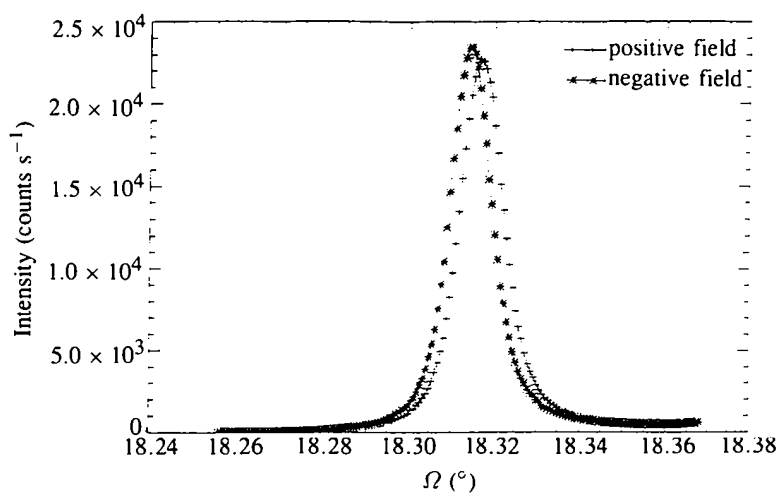

(a)

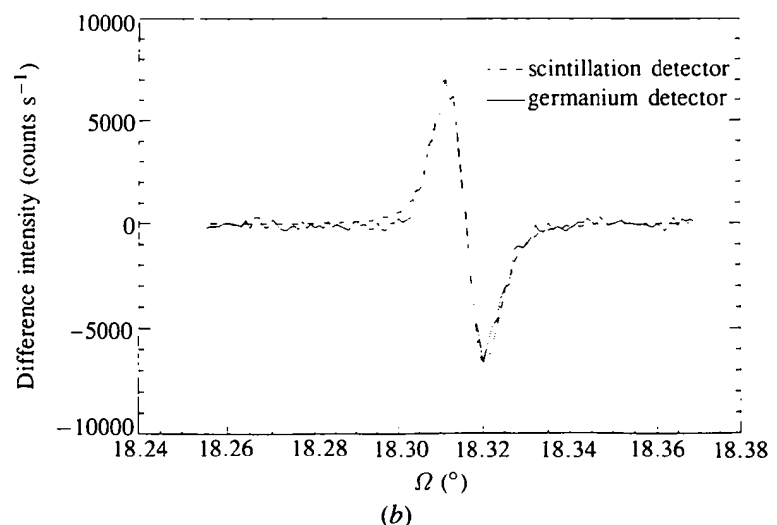

Figure 5

Influence of an external electric field on the (0012) reflection of $\mathrm{LiNbO}_{3}$. (a) The two profiles corresponding to a positive and a negative field, determined with a scintillator detector and homebuilt synchronization electronics. $(b)$ The difference between the two profiles in ( $a$ ) (dashed line) and difference profile determined directly with the germanium detector and lock-in amplifier (solid line). profile. Since the absorption of the Si diode is very small at energies above $25 \mathrm{keV}$, the signal is not influenced significantly.

\section{Conclusions}

The tested 403HS germanium detector has shown to be linear both in a low-flux counting-mode operation $(<1$ to $10^{5}$ photons $\mathrm{s}^{-1}$ ) and in a high-flux current-mode operation (equivalent count rate up to $10^{9}$ photons $\mathrm{s}^{-1}$ ). The dead time in photon-counting mode is $1.9 \mu \mathrm{s}$, which is comparable with a standard $\mathrm{NaI}$ scintillator detector. There is sufficient overlap between the counting and current mode for scaling the two ranges together. This is especially true when the current mode is used in conjunction with a chopped signal source and synchronous lock-in detection, in which case signals below $10^{3}$ photons $\mathrm{s}^{-1}$ can be measured for signal integration times of $300 \mathrm{~ms}$. The detector has a time response in the order of $1 \mu \mathrm{s}$, making it a suitable detector for perturbation measurements in general and for crystals in electric fields in particular.

Ideally, the detector should incorporate two preamplifiers. An electrometer optimized for low drift and low noise for current-mode operation with slowly varying signals, and a 'charge' preamplifier suitable for post amplification by a high-rate nuclear spectroscopy amplifier incorporating baseline correction for rapidly varying signals. This last option should be used cither in counting mode for weak X-ray fluxes, or in current mode at high fluxes using the lock-in amplifier technique to compensate for d.c. drift. The present detector provides a compromise solution between these two ideals, offering a low-price system of wide dynamic range.

It is a pleasure to thank $\AA$ ke Kvick for his continuous support and discussions during the project.

\section{References}

Chipman, D. R. (1969). Acta Cryst. A25, 209-213.

Fujimoto, I. (1982). Acta Cryst. A38, 337-345.

Graafsma, H., Paturle, A., Wu, L., Sheu, H.-S., Majewski, J., Poorthuis, G. \& Coppens, P. (1992). Acta Cryst. A48, 113-120. Jemian, P. R. (1990). J. Appl. Cryst. 23, 430-432.

Kvick, Å. \& Wulff, M. (1992). Rev. Sci. Instrum. 63, 1073-1076.

Paturle, A., Graafsma, H., Sheu, H.-S., Coppens, P. \& Becker, P. (1991). Phys. Rev. B, 43, 14683-14691.

Ståhl, K., Kvick, Å. \& Abrahams, S. C. (1990). Acta Cryst. A46, $478-485$.

Storb, Ch., Dedek, U., Weber, W., Lengeler, B. \& Schuster, M. (1991). Nucl. Instrum. Methods, A306, 544-548. 